\title{
Postoperative cerebral glucose metabolism in pediatric patients receiving proton therapy for craniopharyngioma
}

\author{
Chiaho Hua, PhD, ${ }^{1}$ Barry L. Shulkin, MD, ${ }^{1}$ Daniel J. Indelicato, MD, ${ }^{2}$ Yimei Li, PhD, ${ }^{3}$ Xingyu Li, MS, ${ }^{3}$ \\ Frederick A. Boop, MD, ${ }^{4}$ and Thomas E. Merchant, DO, PhD ${ }^{1}$ \\ Departments of ${ }^{1}$ Radiological Sciences and ${ }^{3}$ Biostatistics, St. Jude Children's Research Hospital; ${ }^{4}$ Semmes-Murphey Neurologic \\ and Spine Institute, Memphis, Tennessee; and 'Radiation Oncology, University of Florida, Jacksonville, Florida
}

\begin{abstract}
OBJECT The aim of the study was to document postoperative cerebral glucose distribution before proton therapy using F-18 fluorodeoxyglucose positron emission tomography (FDG PET) in children with craniopharyngioma.

METHODS Between August 2011 and April 2014, 50 patients (20 males, 30 females) enrolled in a prospective trial for craniopharyngioma underwent FDG PET imaging before proton therapy. Proton therapy was delivered using doublescattered beams with a total prescribed dose of 54 cobalt gray equivalent. Tracer uptake in each of 63 anatomical regions was computed after warping PET images to a 3D reference template in Talairach coordinates. Regional uptake was deemed significantly low or high if it exceeded age-corresponding $95 \%$ prediction intervals of the normal population. The reference group included 132 children with non-CNS-related diseases and normal-appearing cerebral FDG PET scans.
\end{abstract}

RESULTS Median patient age at diagnosis was 8.5 years (range 2-18 years). Forty-eight patients underwent 1-4 tumor-related surgeries before proton therapy, including placement of an Ommaya reservoir in 14 patients. Sixteen patients had symptomatic hydrocephalus that was treated with temporary (external ventricular drain, $n=16$ ) or permanent CSF shunting (ventriculoperitoneal shunt, $n=1$ ). The most commonly seen PET abnormalities in patients before proton therapy were significantly reduced uptake in subregions of the frontal lobe (often involving more than 1 gyrus), medial and ventral portions of the temporal lobe, cingulate gyrus, and caudate nucleus. A significantly high uptake was frequently observed on the contralateral side, including the superior, medial, and inferior temporal gyri and a large portion of the parietal lobe. Statistically significant predictor variables identified in the multivariate analysis for the extent of hypometabolism were sex $(p=0.005)$, hydrocephalus $(p=0.026)$, and the number of tumor-related surgeries $(p=0.017)$.

CONCLUSIONS Postoperative FDG PET of patients with craniopharyngioma revealed metabolic abnormalities in specific regions of the brain. The ability to identify anatomical metabolic defects in individual patients facilitates the investigation of brain injury in children with craniopharyngioma.

Clinical trial registration no.: NCT01419067 (clinicaltrials.gov)

http://thejns.org/doi/abs/10.3171/2015.4.PEDS159

KEY WORDS craniopharyngioma; surgery; proton therapy; positron emission tomography; oncology

$\mathrm{T}$ HE prognosis for children with craniopharyngioma treated with limited surgery and radiation therapy is excellent. ${ }^{7}$ Progression-free and overall survival rates of $77 \%$ and $83 \%$, respectively, at 10 years were reported 2 decades ago in a benchmark series by Rajan et al. ${ }^{16}$ A more recent study estimated progression-free survival to be as high as $96 \%$ when measured at 5 years. ${ }^{9}$ Despite this success, survivors continue to experience neurobehavioral, emotional, and social problems. , $^{42,17,18}$
Considerable effort has been made to reduce treatmentinduced sequelae. For unfavorable tumor localization, limited resection followed by focal irradiation is recommended in lieu of radical resection to better preserve the hypothalamus, pituitary, and optic pathways. ${ }^{11,15}$ With improved endoscopic instrumentation and image guidance systems, the indications for less invasive surgery, including transsphenoidal surgical approaches, continue to expand, and lower morbidity is expected. ${ }^{5,19}$ Proton therapy is also

ABBREVIATIONS CGE = cobalt gray equivalent; EVD = external ventricular drain; FDG PET = F-18 fluorodeoxyglucose positron emission tomography; SUV = standard uptake value; VP = ventriculoperitoneal.

SUBMITTED January 6, 2015. ACCEPTED April 24, 2015.

INCLUDE WHEN CITING Published online August 21, 2015; DOI: 10.3171/2015.4.PEDS159. 
being tested in clinical trials because of its ability to spare normal tissues from radiation exposure as compared with conventional methods of radiation therapy using x-rays. ${ }^{1,8}$

Late effects in survivors with craniopharyngioma are traditionally assessed by clinical examinations, laboratory tests, anatomical MRI, and neuropsychological evaluations. Although functional imaging such as PET may be performed to monitor tumor response and detect recurrence in a variety of brain tumor systems, its role in the evaluation of craniopharyngioma is unknown.

In this study, we investigated the effects of surgery on the brain of children with craniopharyngioma using F-18 fluorodeoxyglucose positron emission tomography (FDG PET). Regions of metabolic abnormality after surgery were identified and monitored after proton therapy to observe for changes in brain metabolism. The long-term goal is to understand the mechanism of treatment-related sequelae in survivors of craniopharyngioma and design the treatment plans for future patients to minimizing such effects.

\section{Methods}

\section{Patient Population and Imaging Studies}

From August 2011 to April 2014, 50 patients with craniopharyngioma were enrolled in a prospective trial and treated with proton therapy (ClinicalTrials.gov identifier: NCT01419067). The trial was approved by the institutional review board at St. Jude Children's Research Hospital. The prerequisites for proton therapy were diagnosis of craniopharyngioma and residual disease after surgery. The protocol specified FDG PET examinations at the time of study entry and at 18 months and 36 months after the start of proton therapy. All 50 patients included in the analysis underwent FDG PET scans prior to therapy. Written informed consent was obtained per institutional policy.

\section{Surgery and Proton Therapy}

Surgery was performed as required prior to proton therapy. When gross-total resection was not deemed feasible, surgery was limited to decompressing vital structures, improving neurological symptoms, or relieving hydrocephalus. The gross tumor volume (GTV) for proton therapy was defined based on multiplanar, multisequence, pre- and postoperative MRI and included the solid and cystic components of the tumor. The protocol specified a 5-mm anatomically constrained clinical target volume (CTV) margin. Proton therapy was delivered at the University of Florida Health Proton Therapy Institute using the double-scattered method. The total prescribed dose of radiation was 54 cobalt gray equivalents (CGE) administered in 30 fractions. A representative dose distribution is shown in Fig. 1. Weekly MRI was performed to monitor for cystic expansion during proton therapy. Treatment plans were modified when the redefined GTV expanded to approach the previously defined CTV.

\section{PET Image Acquisition and Analysis}

FDG PET studies were performed, with patients instructed to fast for at least 4 hours prior to scanning. The preinjection blood glucose level was less than $120 \mathrm{mg} / \mathrm{dl}$ in each patient. Radiopharmaceutical dose was $0.15 \mathrm{mCi} /$ $\mathrm{kg}$ body weight $(2 \mathrm{mCi}$ minimum per dose and $12 \mathrm{mCi}$ maximum). Emission data of the brain were acquired in 3D mode for 8 minutes, 1 hour after the administration of the FDG, using a GE Discovery 690 PET/CT system (GE Medical Systems) with attenuation correction applied from the accompanying low-dose CT. Using HERMES Brain Analysis software version 3.5 (Hermes Medical Solutions), FDG PET images of patients were nonlinearly warped to a 3D reference template in Talairach coordinates created from normal subjects. Since the absolute standard uptake value (SUV) is subject to many sources of variability, ${ }^{2}$ we normalized the count per voxel in each of the 63 anatomical regions by the average count per voxel in all regions to facilitate comparisons between patients. This measure is called the uptake ratio, which is identical to the SUV ratio. The list of anatomical regions was summarized in a previous paper ${ }^{10}$ and consists of various gyri of the frontal lobes, parietal lobes, temporal lobes, occipital lobes, and the limbic system, as well as the caudate nucleus, putamen, thalamus, cerebellum, and brainstem. The regional uptake ratio was deemed significantly low or high when its value exceeded age-corresponding $95 \%$ prediction intervals for the reference population. Results for a stricter threshold of $99 \%$ were also reported.

\section{Reference Group for Comparison}

Since healthy children rarely undergo PET scans, we used the cerebral FDG distribution of 132 patients (68 males and 64 females) aged 1-20 years with non-CNS diseases as a surrogate for the normal population for comparison with craniopharyngioma patients. The use of oncology patients as a normal surrogate is a limitation of our study. Primary diagnoses in this population included Hodgkin lymphoma (33 cases), non-Hodgkin lymphoma (20), osteosarcoma (10), Ewing sarcoma (8), melanoma (5), and a variety of other tumors (56). These PET scans, acquired between August 2011 and July 2013, were reviewed by an experienced nuclear medicine physician to ensure normal cerebral distribution and selected only if they were performed before the administration of any systemic chemotherapy and anticancer drugs. No prior radiation treatments were given to these reference subjects. Each reference subject contributed 1 PET dataset.

The brain section of the whole-body PET scan was processed following the same aforementioned procedure. For each anatomical region, scatter plots of regional uptake ratio versus age were fitted with linear and spline (piecewise linear) models. The best model with the smallest Akaike information criterion was chosen. For each region, age-corresponding $95 \%$ and $99 \%$ prediction intervals of uptake ratio were estimated as reference ranges for subsequent use by craniopharyngioma patients.

\section{Risk Factor Analysis}

Simple and multiple negative binomial regression analyses were performed to identify statistically significant $(\mathrm{p}<$ $0.05)$ predictor variables for the extent of hypometabolism on baseline pre-irradiation PET scans. Risk factors with borderline significance $(p<0.1)$ in simple negative bino- 


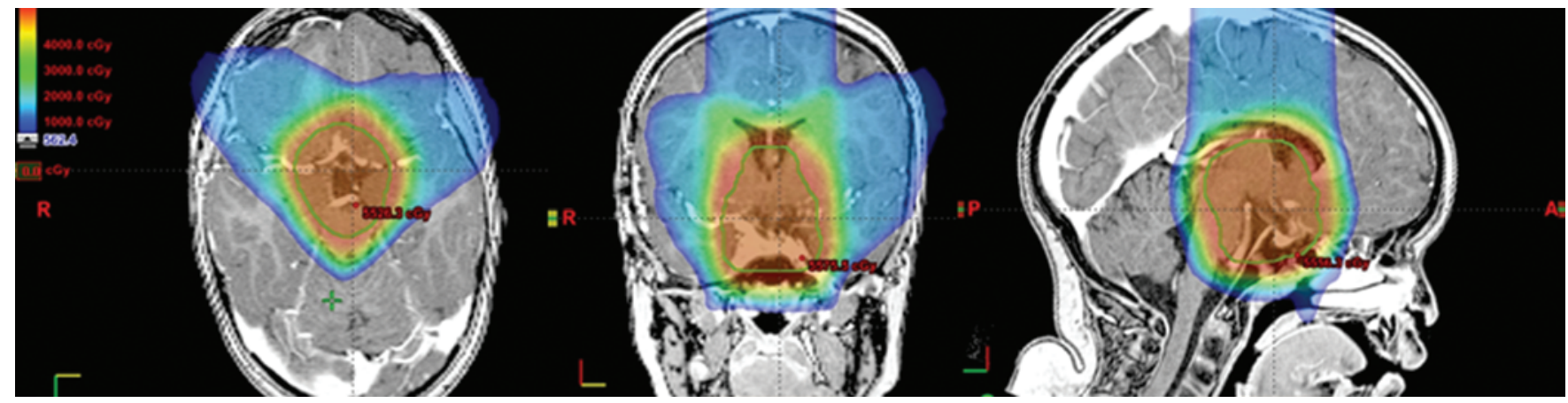

FIG. 1. Three overlaid orthogonal T1-weighted MR images showing dose distributions in a patient irradiated with 3 proton beams. The green contours are the planning target volume. Red, green, and blue colors in the color-washed dose distribution represent high, medium, and low doses (approximately 54, 30, and 10 CGE, respectively). Figure is available in color online only.

mial regression analysis were included for further evaluation with multiple negative binomial regression analysis. All statistical analyses were performed using SAS 9.3 software. For this analysis, we used $99 \%$ prediction intervals as a strict threshold to define abnormality. Clinical variables tested include sex (male vs female), maximum tumor dimension on MRI at diagnosis, hydrocephalus, Ommaya reservoir placement, CSF diversion, age at the first surgery, surgical approach (no surgery or only transsphenoidal surgery vs craniotomy), number of tumor-related surgeries, and number of cranial surgeries. The time since last surgery to baseline PET was also tested.

\section{Results}

\section{Patient Characteristics}

The median age at diagnosis for the 50 patients (20 males, 30 females) was 8.5 years (range $2-18$ years). Two patients did not have surgery prior to the PET evaluation and proton therapy. Sixty-nine tumor-related surgeries and 19 non-tumor-related surgeries were recorded for the remaining 48 patients before proton therapy. Tumor-related surgical approaches included 41 craniotomy (34 with resection, 4 with Ommaya catheter placement without tumor resection, 3 with Ommaya catheter placement and tumor resection); 9 transsphenoidal resection (6 with endoscope, 3 without endoscope); and 15 bur hole (5 with Ommaya catheter placement using image guidance and 3 using endoscope, 4 endoscopic cyst fenestration, 2 endoscopic resection with external ventricular drain [EVD] placement, 1 with endoscopic resection). Additional tumor-related procedures recorded as revisions included Ommaya catheter revision $(n=2)$ or removal $(n=2)$. All revision procedures used existing sites of intracranial access.

Non-tumor-related procedures prior to proton therapy included bur hole followed by placement of EVD $(n=13)$ or ventriculoperitoneal (VP) shunt to treat hydrocephalus, bur hole access to evacuate postoperative hematoma $(\mathrm{n}=$ 1 ) or drainage of subgaleal fluid $(n=1)$. One patient had a history of intracranial bolt placed after an episode of head trauma. Two non-tumor-related craniotomies were performed for VP shunting $(n=1)$ and evacuation of postoperative hematoma $(n=1)$. Finally, 1 patient underwent conversion of EVD to VP shunt. Seven tumor-related surgeries were performed in 6 patients after the baseline PET evaluation and prior to proton therapy including craniotomy with resection $(n=3)$, craniotomy with Ommaya catheter and reservoir placement $(n=3)$, and craniotomy with VP shunt placement $(n=1)$. One of the patients required an additional procedure to redirect the Ommaya catheter. Although 9 patients underwent transsphenoidal surgery, 6 did not have additional surgery of any type performed prior to the PET evaluation.

After the start of proton therapy, 4 patients required intracranial procedures. One patient underwent craniotomy with resection for postirradiation cyst progression and subsequently required bur hole drainage of subdural fluid; 1 patient underwent craniotomy with endoscopic resection and subsequently had his VP shunt converted to an Ommaya reservoir; 1 patient required bur hole for placement of VP shunt; and 1 patient required craniotomy with tumor resection for cyst progression.

Most common procedures performed in all patients were insertion of a ventricular catheter with an external drainage device (16 patients) and the placement of an Ommaya reservoir (14 patients). Hydrocephalus was present in 24 patients.

The median time from the last surgery to the baseline PET study was 78 days (range 7-1361 days) for 43 patients undergoing surgery before baseline PET studies. The median time from the baseline PET to the start of proton therapy for all patients was 24 days (range 1-88 days).

\section{Patient PET Findings}

The most remarkable finding was globally decreased FDG uptake in the unilateral hemisphere corresponding to the side of surgical intervention. Two example cases with predominantly right-side PET abnormalities are shown in Fig. 2. In the first case, the patient underwent transcortical craniotomy with resection and EVD, craniotomy with evacuation of hematoma, and endoscopic cyst fenestration prior to evaluation with PET. After the PET study and prior to proton therapy, the patient underwent bifrontal transcallosal craniotomy with partial resection. In the second case, the patient underwent transsphenoidal surgery followed by Ommaya reservoir placement and subsequent revision.

Table 1 summarizes the number and location of significantly low and high glucose metabolism before proton therapy in the 50 study patients. The most commonly seen 

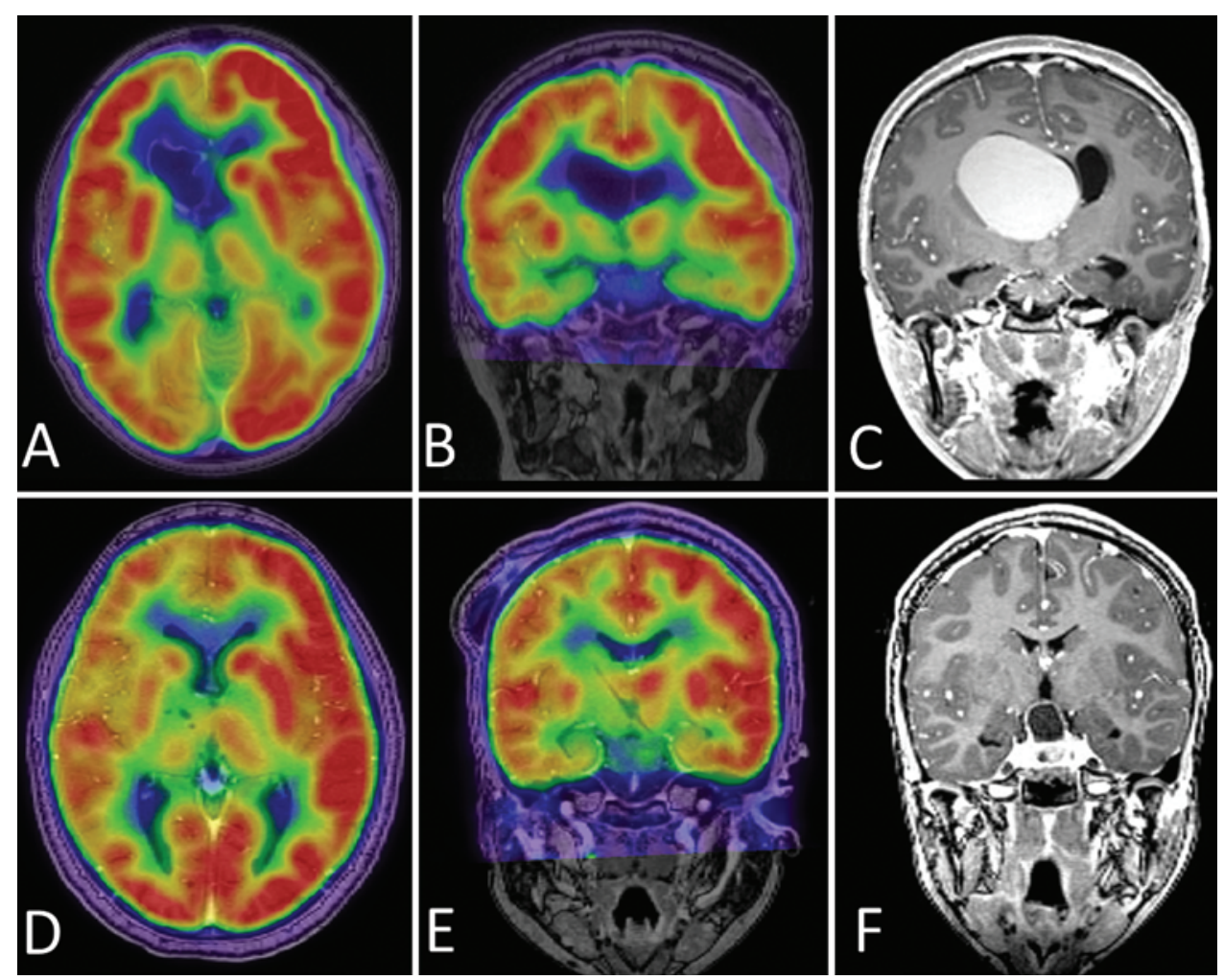

FIG. 2. Images obtained in 2 patients with craniopharyngioma whose baseline PET scans show significant postoperative asymmetry of FDG uptake. The PET rainbow color scale was adopted, with red representing higher uptake and purple representing lower uptake. A-C: Axial (A) and coronal (B) PET scans and a preoperative coronal contrast-enhanced T1-weighted MR image (C) obtained in a 9 -year-old girl who showed $22 \%, 26 \%$, and $14 \%$ lower uptake on the right side than the left side in the superior, medial, and inferior frontal gyri, respectively. D-F: Axial (D) and coronal (E) PET scans and a preoperative coronal contrastenhanced T1-weighted MR image (F) obtained in a 7-year-old girl who showed 15\%,21\%, and 20\% lower uptakes on the right for the superior, medial, and inferior frontal gyri, respectively. Uptakes in the caudate nucleus, putamen, and thalamus were approximately $20 \%$ lower on the right. Figure is available in color online only.

PET abnormality was significantly reduced uptake in subregions of the frontal lobe, medial and ventral portions of the temporal lobe (uncus, hippocampus, fusiform), cingulate gyrus, and caudate nucleus. The frontal lobe abnormalities often involved more than 1 gyrus.

Uptake in the cerebellum, brainstem, putamen, precentral and postcentral gyri, and occipital lobe was less affected. The majority of abnormalities in the frontal lobe, caudate nucleus, and thalamus were unilateral corresponding to the side of intervention. As expected, most of the midline tumors were approached on the side of the nondominant (right) hemisphere. On the contrary, a significantly high uptake was frequently observed on the contralateral side, including the superior, medial, and inferior gyri of the temporal lobe and a large portion of the parietal lobe, including lobules par superior and inferior, gyrus supramarginalis, and gyrus angularis.

\section{Risk Factor Analysis}

Simple negative binomial regression analysis identified statistically significant risk factors for the extent of hypometabolism, including sex $(\mathrm{p}=0.009)$, hydrocephalus ( $\mathrm{p}$ $=0.045)$, and the number of tumor-related surgeries ( $\mathrm{p}=$ $0.028)$. The number of cranial surgeries had a marginal significance $(p=0.0506)$. The time since last surgery to baseline PET was not significant $(\mathrm{p}=0.541)$. Only sex ( $\mathrm{p}$
$=0.005)$, hydrocephalus $(\mathrm{p}=0.026)$, and the number of tumor-related surgeries $(\mathrm{p}=0.017)$ remained significant in multiple negative binomial regression analysis results. $\mathrm{Fe}-$ male patients and patients with hydrocephalus or receiving more tumor surgeries were at higher risk of showing FDG PET hypometabolism in the brain before proton therapy.

\section{Discussion}

Approximately one-third of our patients showed metabolic abnormalities in multiple cerebral regions on FDG PET scans after surgery but before proton therapy. Hypometabolism was detected in the frontal lobes, medial and inferior temporal lobes, limbic system, caudate nuclei, and thalamic nuclei. There was a strong association with female sex, which echoes the findings that female sex was an independent predictor of increased long-term cardiovascular, neurological, and psychosocial morbidity in craniopharyngioma patients. ${ }^{14}$ Hydrocephalus from mass effect and more tumor surgeries also contributed to the extent of abnormality. Since most patients received surgery prior to enrollment, preoperative PET scans were not available for comparison. Therefore, it was difficult in some regions to ascertain the relative contributions of tumor and surgical effects.

Potential sources of PET abnormality observed before 
TABLE 1. The numbers of patients with significantly low and high cerebral FDG uptake on baseline PET before proton therapy $(n=50)^{*}$

\begin{tabular}{|c|c|c|c|c|c|c|}
\hline \multirow[b]{2}{*}{ Anatomical Region } & \multicolumn{3}{|c|}{ 95\% Criterion } & \multicolumn{3}{|c|}{$99 \%$ Criterion } \\
\hline & Normal & Low & High & Normal & Low & High \\
\hline Cerebellum & 41 & 7 & 2 & 47 & 2 & 1 \\
\hline \multicolumn{7}{|l|}{ Frontal lobe } \\
\hline Gyrus frontalis superior, medius, \&/or inferior† & 18 & 16 & 27 & 27 & 15 & 19 \\
\hline Gyrus frontalis superior pars medius & 37 & 11 & 2 & 40 & 8 & 2 \\
\hline Gyrus rectus \&/or orbitalis & 34 & 14 & 2 & 40 & 10 & 0 \\
\hline Gyrus precentralis $†$ & 28 & 4 & 20 & 31 & 3 & 18 \\
\hline \multicolumn{7}{|l|}{ Parietal lobe } \\
\hline Gyrus postcentralis $\dagger$ & 29 & 3 & 20 & 37 & 1 & 13 \\
\hline $\begin{array}{l}\text { Lobules par superior \& inferior, gyrus supramarginalis, } \\
\quad \& / \text { or gyrus angularis } \dagger\end{array}$ & 12 & 10 & 31 & 25 & 6 & 22 \\
\hline Precuneus & 36 & 8 & 6 & 42 & 5 & 3 \\
\hline \multicolumn{7}{|l|}{ Occipital lobe } \\
\hline Cuneus & 36 & 11 & 3 & 45 & 4 & 1 \\
\hline Gyrus occipitalis superior, medius, \&/or inferior† & 27 & 8 & 18 & 36 & 7 & 7 \\
\hline \multicolumn{7}{|l|}{ Temporal lobe } \\
\hline Gyrus temporalis superior, medius, \&/or inferior† & 19 & 8 & 26 & 26 & 6 & 18 \\
\hline Uncus, hippocampus, \&/or fusiform $†$ & 22 & 23 & 7 & 33 & 12 & 5 \\
\hline Limbic system, gyrus cinguli & 31 & 17 & 2 & 34 & 14 & 2 \\
\hline Thalamus & 36 & 14 & 0 & 44 & 6 & 0 \\
\hline Putamen & 43 & 5 & 2 & 48 & 2 & 0 \\
\hline Caudate nuclei & 29 & 19 & 2 & 34 & 15 & 1 \\
\hline Brainstem & 42 & 5 & 3 & 46 & 2 & 2 \\
\hline Insula† & 36 & 11 & 6 & 40 & 7 & 4 \\
\hline
\end{tabular}

* The sum of the numbers of patients with normal, low, and high uptakes in some regions may exceed 50.

$\dagger$ Indicates regions where some patients had both significantly low and high glucose uptakes, with low on one side and high on the other.

proton therapy included caudate and thalamic infarction due to occlusion of perforating arteries, cyst catheter tracts extending through tissues, surgical defects in frontal lobes as indicated by MRI, and vision-related deficits in occipital gyri, cuneus, and fusiform gyri. Abnormality in straight gyri (gyrus rectus and orbitalis), mostly detected on the right side, may be related to frontal lobe retraction in right frontal and pterional craniotomies. On the other hand, hypometabolism in both cingulate gyri was as common as unilateral abnormality. While direct injury from transcallosal surgery may explain some occurrences, many could result from indirect effects from damage to components of the limbic system. Brain tissue deformation due to mass effect and obstructive hydrocephalus of lateral and third ventricles were frequently seen on preoperative MRI. Although many resolved automatically by the time of our baseline PET studies, which were performed on average 3 months after surgery, associated metabolic abnormalities may persist. For example, significant midbrain and pons deformation by tumor was present at diagnosis in 5 of 7 patients with subsequent abnormal FDG uptake in the brainstem (hypometabolism in 5 cases and hypermetabolism in 2).

Metabolic hyperactivity was detected on baseline PET images in the parietal lobe, the temporal lobe, and to a lesser extent, the frontal lobe. Most of them, involving multiple regions, were in the hemisphere contralateral to the surgical and procedural side. This finding has not been previously reported in the literature for patients with craniopharyngioma. It is well known that the caudate nucleus, putamen, and thalamus are essential components of the corticostriatal circuit. The cerebral cortex sends excitatory projections to caudate nucleus and putamen while receiving inputs from thalamus. We speculate that our observation could reflect a compensatory response to the injury to thalamus and striatum. In fact, all patients who had hypermetabolism in the parietal lobe also showed hypometabolism in striatum, thalamus, or both in the contralateral hemisphere. The clinical significance of such neural reorganization in patients with craniopharyngioma is yet to be determined.

Effects of radiation therapy on the brain require longterm follow-up to assess the clinical impact. This is particularly important for new technologies such as proton therapy. The areas outside the target exposed to low and medium doses are dramatically reduced compared with conventional photon irradiation. Our PET scans acquired 18 months after proton therapy for the initial 17 patients indicated that the number of metabolic abnormalities is in general reduced. Multiple abnormalities were still present in a quarter of the patients. Figure 3 shows 2 example patients. Detecting persistent and new hypometabolic re- 
gions may require quantitative comparison with normal benchmark data. Many metabolic defects were bilaterally symmetrical and the difference in uptake from reference regions used by human observers, such as cerebellum and contralateral white matter, may be too small for qualitative recognition.

It was hypothesized that injury to the hypothalamus in addition to the direct operative trauma to the frontal lobe may cause behavior problems in craniopharyngioma patients. ${ }^{3}$ Hypothalamic damage has also been shown to remotely impact the efficiency of memory retrieval in medial prefrontal cortex. ${ }^{13}$ In our study, unilateral metabolic defects were confirmed in specific regions of the frontal lobe, as shown in Table 1, on the surgical and procedural side. Correlation with cognitive, emotional, and behavioral symptoms will allow us to determine the significance of persistent frontal lobe hypometabolism after treatment. However, assessment of the metabolic activity of the hypothalamus in children is problematic due to low FDG avidity and partial volume effect from low spatial resolution of PET images. Therefore, direct injury to the hypothalamus may still need to be assessed based on anatomical MRI, endocrine functions, and autonomics. In a patient who had a small tumor with bilateral hypothalamic involvement and received only transsphenoidal surgery without invasive procedures, hypometabolism was seen in bilateral
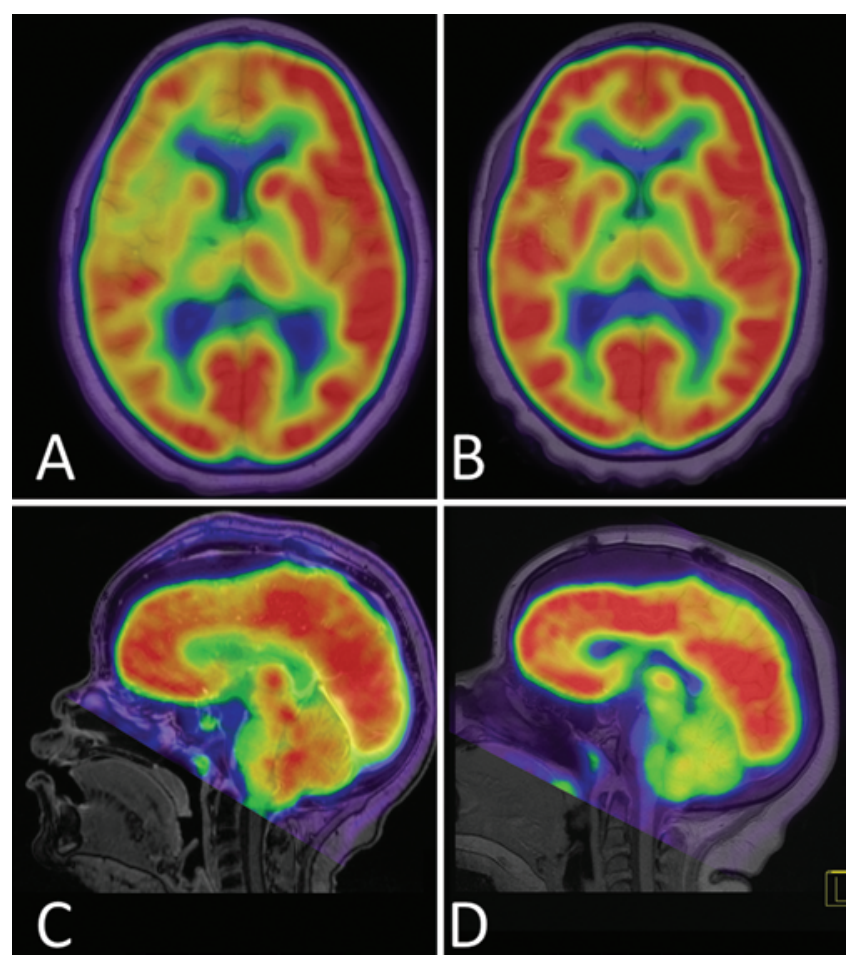

FIG. 3. A and B: Axial PET scans obtained in a 7-year-old girl showing an improvement in the right hemisphere from the time after surgery $(A)$ to 18 months after proton therapy (B). Uptake in the right hemisphere was approximately $20 \%$ lower than the left after surgery, and the difference was $<10 \%$ at 18 months after proton therapy. C and D: Sagittal PET scans obtained in a 7-year-old girl showing an uptake reduction in several areas, including thalamus, brainstem, and cerebellum from the time after surgery $(C)$ to 18 months after proton therapy $(D)$. The reduction in uptake ratio was $27 \%, 37 \%$, and $34 \%$ in the thalamus, brainstem, and cerebellum, respectively. Figure is available in color online only. gyrus rectus, hippocampus, fusiform, and insula before proton therapy. This observation likely reflects the functional connectivity of the hypothalamus with the prefrontal cortex and many components in the limbic circuitry. In patients without direct operative trauma to the frontal lobe and the rest of the limbic system, it is reasonable to focus attention on the hypothalamus. However, radiation effects on the entire limbic system should be investigated as well because the limbic system plays important roles in motivation, emotion, learning, and memory, and many components still receive a prescribed high tumoricidal dose even with proton therapy. Some speculated that cognitive impairment could be caused by craniopharyngioma itself interrupting the reciprocal projections between the frontal lobes and the hypothalamus. ${ }^{6}$ This may require tractography of diffusion tensor imaging to determine if such injury, once occurred, would recover after surgical removal of the mass.

\section{Conclusions}

This study identified specific cerebral regions of hypometabolism in pediatric craniopharyngioma patients receiving surgery and proton therapy. Metabolic abnormalities were present before proton therapy and occurred more often in female patients and those with hydrocephalus or receiving multiple tumor surgeries. The ability to identify abnormal regions of cerebral metabolism in individual patients may facilitate personalized treatment plan adaptation and the investigation of neurotoxicity in children with craniopharyngioma.

\section{Acknowledgments}

We wish to thank Anne Madey and Tina Davis for their contribution in data collection and clinical trial management, Lisa Mills for PET software assistance, and Roletta Ammons for manuscript editing.

\section{References}

1. Boehling NS, Grosshans DR, Bluett JB, Palmer MT, Song X, Amos RA, et al: Dosimetric comparison of three-dimensional conformal proton radiotherapy, intensity-modulated proton therapy, and intensity-modulated radiotherapy for treatment of pediatric craniopharyngiomas. Int J Radiat Oncol Biol Phys 82:643-652, 2012

2. Boellaard R: Standards for PET image acquisition and quantitative data analysis. J Nucl Med 50 (Suppl 1):11S-20S, 2009

3. Cavazzuti V, Fischer EG, Welch K, Belli JA, Winston KR: Neurological and psychophysiological sequelae following different treatments of craniopharyngioma in children. J Neurosurg 59:409-417, 1983

4. Dolson EP, Conklin HM, Li C, Xiong X, Merchant TE: Predicting behavioral problems in craniopharyngioma survivors after conformal radiation therapy. Pediatr Blood Cancer 52:860-864, 2009

5. Elliott RE, Jane JA Jr, Wisoff JH: Surgical management of craniopharyngiomas in children: meta-analysis and comparison of transcranial and transsphenoidal approaches. Neurosurgery 69:630-643, 2011

6. Honegger J, Barocka A, Sadri B, Fahlbusch R: Neuropsychological results of craniopharyngioma surgery in adults: a prospective study. Surg Neurol 50:19-29, 1998

7. Kiehna EN, Merchant TE: Radiation therapy for pediatric craniopharyngioma. Neurosurg Focus 28(4):E10, 2010 
8. Merchant TE, Hua CH, Shukla H, Ying X, Nill S, Oelfke $\mathrm{U}$ : Proton versus photon radiotherapy for common pediatric brain tumors: comparison of models of dose characteristics and their relationship to cognitive function. Pediatr Blood Cancer 51:110-117, 2008

9. Merchant TE, Kun LE, Hua CH, Wu S, Xiong X, Sanford $\mathrm{RA}$, et al: Disease control after reduced volume conformal and intensity modulated radiation therapy for childhood craniopharyngioma. Int J Radiat Oncol Biol Phys 85:e187e192, 2013

10. Montandon ML, Zaidi H: Quantitative analysis of templatebased attenuation compensation in 3D brain PET. Comput Med Imaging Graph 31:28-38, 2007

11. Müller HL: Consequences of craniopharyngioma surgery in children. J Clin Endocrinol Metab 96:1981-1991, 2011

12. Ondruch A, Maryniak A, Kropiwnicki T, Roszkowski M, Daszkiewicz P: Cognitive and social functioning in children and adolescents after the removal of craniopharyngioma. Childs Nerv Syst 27:391-397, 2011

13. Ozyurt J, Lorenzen A, Gebhardt U, Warmuth-Metz M, Müller HL, Thiel CM: Remote effects of hypothalamic lesions in the prefrontal cortex of craniopharygioma patients. Neurobiol Learn Mem 111:71-80, 2014

14. Pereira AM, Schmid EM, Schutte PJ, Voormolen JH, Biermasz NR, van Thiel SW, et al: High prevalence of long-term cardiovascular, neurological and psychosocial morbidity after treatment for craniopharyngioma. Clin Endocrinol (Oxf) 62:197-204, 2005

15. Puget S: Treatment strategies in childhood craniopharyngioma. Front Endocrinol (Lausanne) 3:64, 2012

16. Rajan B, Ashley S, Gorman C, Jose CC, Horwich A, Bloom $\mathrm{HJ}$, et al: Craniopharyngioma-a long-term results following limited surgery and radiotherapy. Radiother Oncol 26:1-10, 1993

17. Waber DP, Pomeroy SL, Chiverton AM, Kieran MW, Scott RM, Goumnerova LC, et al: Everyday cognitive function after craniopharyngioma in childhood. Pediatr Neurol 34:13-19, 2006
18. Zada G, Kintz N, Pulido M, Amezcua L: Prevalence of neurobehavioral, social, and emotional dysfunction in patients treated for childhood craniopharyngioma: a systematic literature review. PLoS ONE 8:e76562, 2013

19. Zona G, Spaziante R: Management of cystic craniopharyngiomas in childhood by a transsphenoidal approach. J Pediatr Endocrinol Metab 19 (Suppl 1):381-388, 2006

\section{Disclosure}

Dr. Indelicato reports being a consultant for Group H and LEK Consulting.

\section{Author Contributions}

Conception and design: Hua, Merchant. Acquisition of data: Shulkin, Indelicato, Boop. Analysis and interpretation of data: Hua, Shulkin Y Li, X Li, Merchant. Drafting the article: Hua, Merchant. Critically revising the article: all authors. Reviewed submitted version of manuscript: Hua, Shulkin, Indelicato, Merchant. Approved the final version of the manuscript on behalf of all authors: Hua. Statistical analysis: Y Li, X Li. Administrative/ technical/material support: Hua, Shulkin, Indelicato, Boop, Merchant. Study supervision: Merchant.

\section{Supplemental Information}

Previous Presentation

Portions of this paper were presented at the American Society for Radiation Oncology 55th Annual Meeting, September 22-25, 2013 , in Atlanta, Georgia.

\section{Correspondence}

Chiaho Hua, Department of Radiological Sciences, St. Jude Children's Research Hospital, 262 Danny Thomas Pl., Mail Stop 220, Memphis, TN 38105-3678. email: chia-ho.hua@stjude.org. 